\title{
Primary Osteogenic Sarcoma of Distal Phalanx of Hand
}

\author{
Sonu Yadav ${ }^{1}$, Uma Sharma², Tanvi Mittal ${ }^{3}$, Sweta ${ }^{4}$
}

\begin{abstract}
Osteosarcoma is rare, accounting for less than $1 \%$ of adult malignancies and $3-6 \%$ at the pediatric age, with peak incidences in adolescence and after 60 years of age. ${ }^{1}$ The incidence of osteosarcoma in the bones of hand has been reported to be low, around $0.9 \% .{ }^{1}$ We report a 37 -year-old female patient who presented with swelling in the distal phalanx of ring finger of left hand since 3 months associated with pain. Imaging studies were consistent with a destructive lesion. Histological examination revealed osteoblastic osteosarcoma of phalanx.
\end{abstract}

Keywords: Osteosarcoma, Osteoblastic

\section{Introduction}

Osteosarcomas are malignant tumors characterized by the direct formation of osteoid or immature bone by malignant cells. ${ }^{2}$ They occur most often in adolescents, peak incidence at the age of 15-19 years during the period of maximum growth. They appear to be related to increased osteoblastic activity with a high tendency for systemic spread. ${ }^{2,3}$ They usually develop in long bones, such as femur, tibia and humerus, showing predilection for metaphyseal areas. ${ }^{2}$ There are various subtypes of osteosarcomas, each with distinct clinical and imaging characteristics. They can be classified as intramedullary (high grade, telengiectatic, low grade, small cell), juxtacortical (parosteal, periosteal, intracortical, high-grade surface), or secondary lesions. ${ }^{4}$

We report a rare presentation of osteoblastic osteosarcoma of distal phalanx of ring finger.

\section{Case Report}

A 37-year-old female patient presented with swelling in ring finger since 3 months, associated with increasing pain. The swelling was hard in consistency. The mass was slightly tender to touch with no signs of inflammation. There was soft tissue swelling around the ring finger, but no periosteal reaction around the lesion.

Past medical history was unremarkable. Laboratory tests were within normal range. MRI shows destructive osseous lesion involving the entire distal phalanx of ring finger with marked extraosseous soft tissue extension; , the lesion however in the soft tissues appears relatively well marginated with T1/T2 hypointense rim around the lesion.

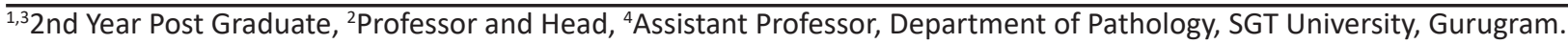

Correspondence: Dr. Sonu Yadav, Department of Pathology, SGT University, Gurugram.

E-mail Id: sonuyadav1992@icloud.com

Orcid Id: http://orcid.org/0000-0002-9126-9579

How to cite this article: Yadav S, Sharma U, Mittal T et al. Primary Osteogenic Sarcoma of Distal Phalanx of Hand. Rec Adv Path Lab Med 2017; 3(3\&4): 4-7.

Digital Object Identifier (DOI): https://doi.org/10.24321/2454.8642.201706

ISSN: $2454-8642$ 


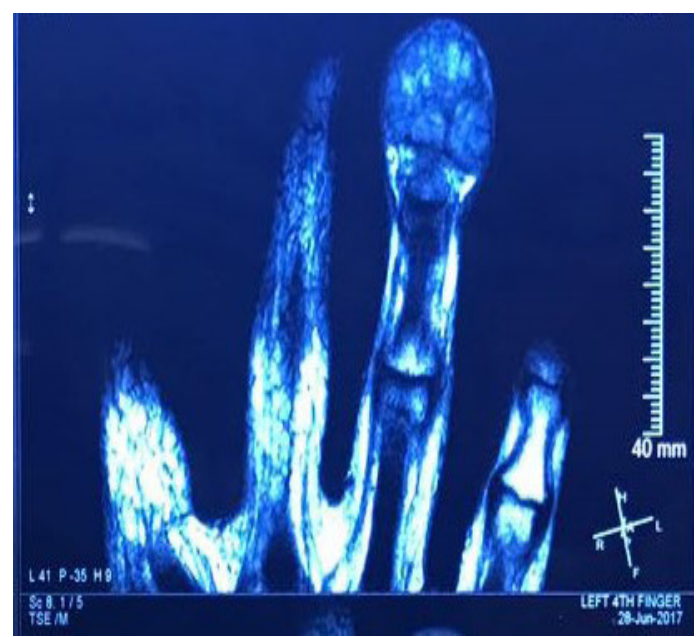

Figure 1.MRI of Left Ring Finger Showing Abnormal Signal Intensity and a Soft Tissue Mass with Inhomogenous High Signal Density

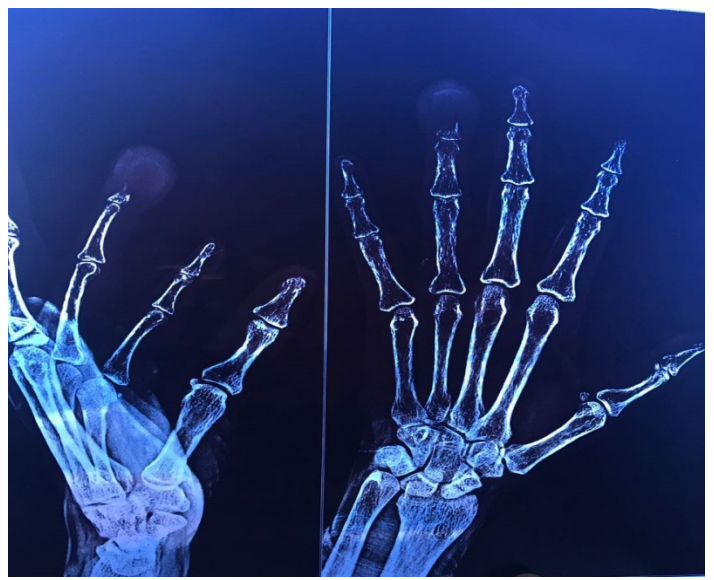

Figure 2.X-ray of Distal Phalanx of Ring Finger Showing Destructive Lesion

Considering the history, physical examination and imaging studies, which were in favor of an aggressive or malignant lesion, surgical excision (amputation) was done for histopathological examination. Grossly we received ring finger measuring $5 \times 2.5 \times 1.5 \mathrm{~cm}$ with a growth at distal end, distal end measuring $2.5 \times 2.5 \mathrm{~cm}$, about $1.5 \mathrm{~cm}$ from resected margin. The cut surface was firm in consistency.

Microscopically showed interlacing fascicles of spindle- shaped cells and low mitotic activity, i.e., 2-3/10 HPF. Numerous tumor giant cells, areas of necrosis, osteoid production were also seen. At places, spindle-shaped cells with marked pleomorphism suggestive of highgrade osteosarcoma, infiltration into adjacent tissue seen. Resected margin was free of tumor.

Histological features were suggestive of osteoblastic osteosarcoma of ring finger.

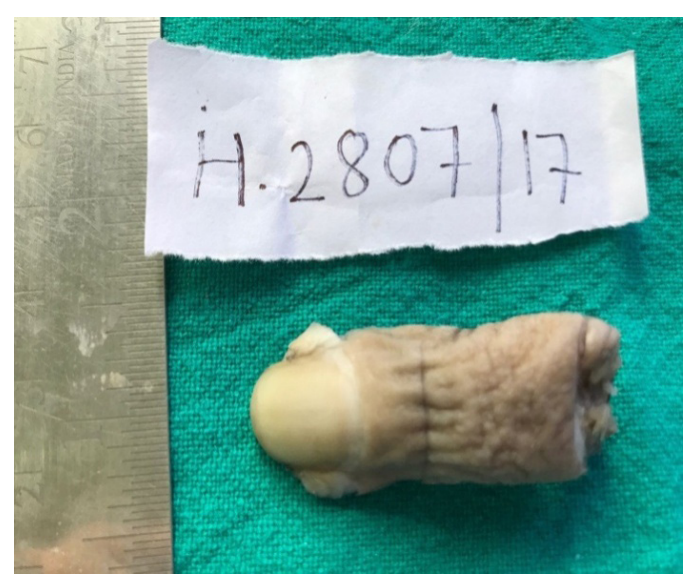

Figure 3.Amputated Ring Finger of Left Hand 


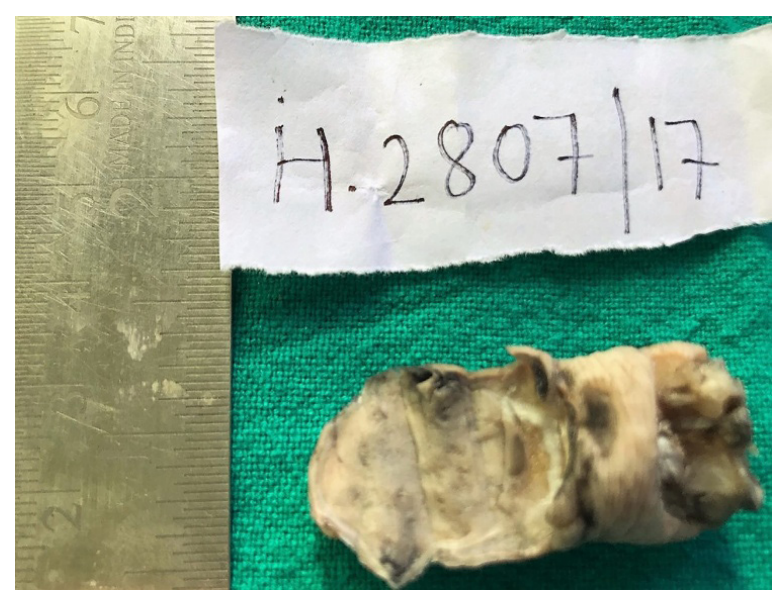

Figure 4.Distal Phalanx of Ring Finger Showing Grey White Tumor Area with Areas of Necrosis and Hemorrhage

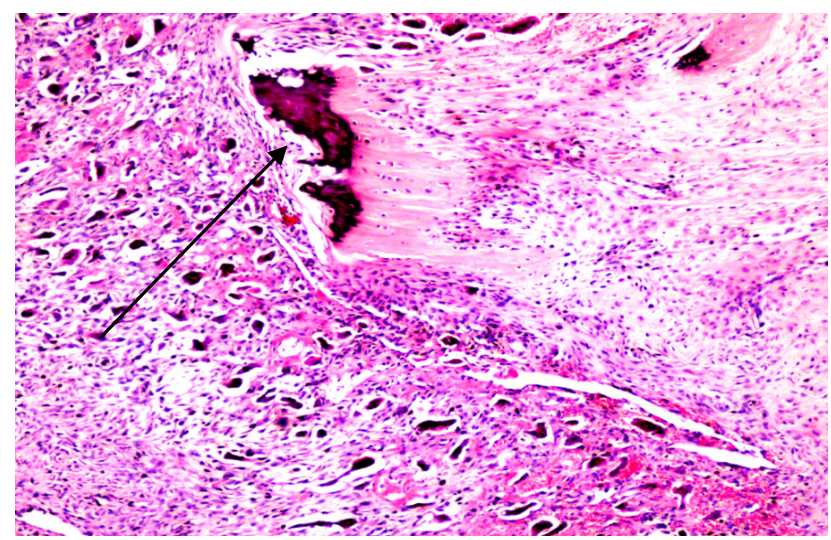

Figure 5.Necrotic Bone $(\uparrow)$ and Pleomorphic Tumor Cells ( $($ )

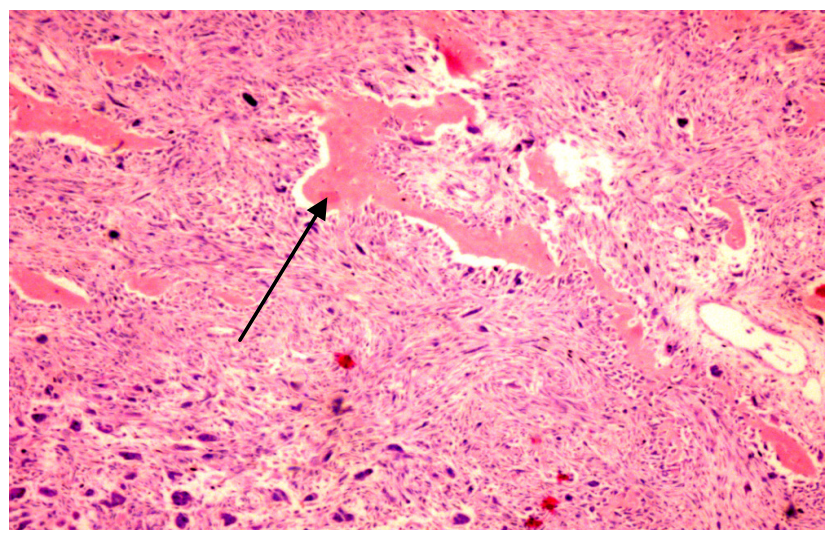

Figure 6.Malignant Tumor Cells Forming Osteoid $(\uparrow)$

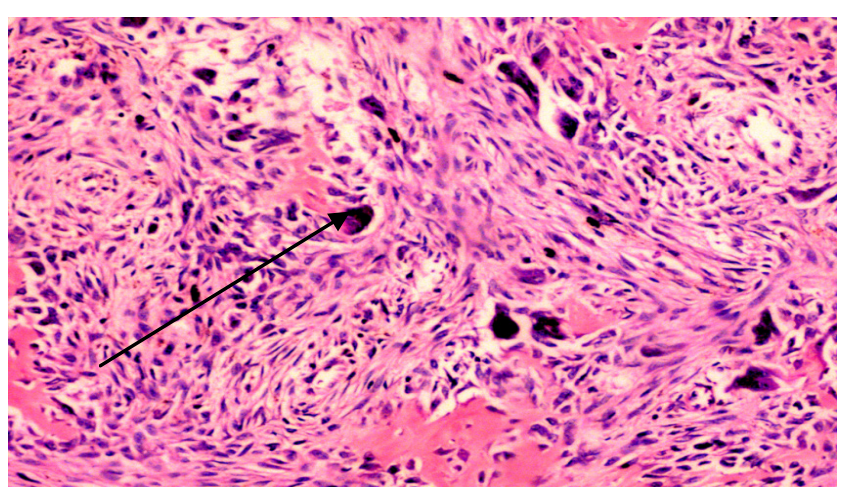

Figure 7.Interlacing Pleomorphic Spindle Shape Cells with Tumor Giant Cells ( $\uparrow)$ 


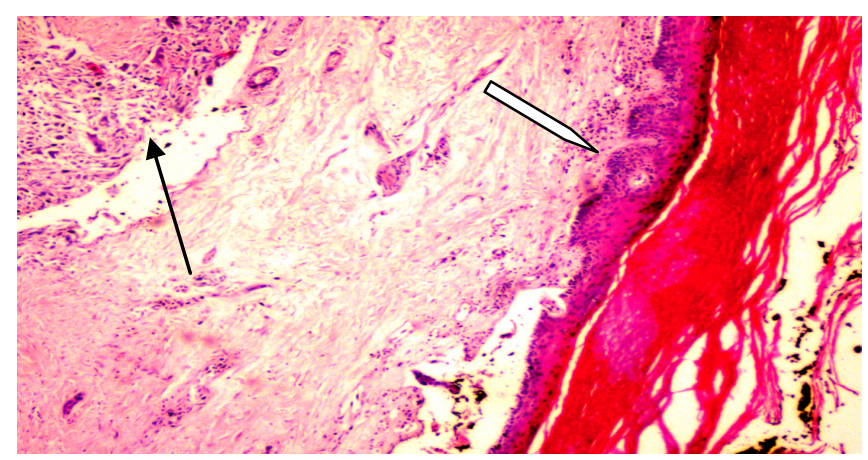

Figure 8.Tumor $(\uparrow)$ beneath the Underlying Skin ( $(\hat{)})$

\section{Discussion}

Osteosarcomas of hands are rare and seemingly these cases differ in presentation and behavior compared to those in usual locations. Patients with osteosarcoma of the hands presents at an age above the fourth decade. This age is higher than the adolescent age, when osteosarcomas of long tubular bones have their peak incidence. ${ }^{1}$ Pain and swelling are the most common presentation similar to our patient. Other symptoms are rare. In small tubular bones, the same symptoms can occur, but since this area is not a common place for osteosarcoma, diagnosis can be difficult without a biopsy. ${ }^{5}$ The differential diagnosis of osteosarcoma includes osteoid osteoma and fibrous dysplasia. ${ }^{2}$ There is predilection for the metacarpo-phalangeal joints with the second metacarpal head being the commonest site of involvement. This correlates with the most active bone growth site in the hand. ${ }^{3}$

The usual MRI findings in osteosarcoma of short tubular bones are similar to those of long bones with cortical destruction and periosteal reaction, together with abnormal marrow signal intensity and peritumoral edema. ${ }^{3}$

Evaluation and treatment of osteosarcoma of the phalanx should be similar to osteosarcoma of the long bones, i.e., amputation. Those with wide and radical margins had no local reccurrence, but those with marginal excision had a considerably high recurrence rate. ${ }^{3}$

A high index of suspicion for osteosarcoma is needed for accurate diagnosis and prompt and proper treatment.

\section{Conclusion}

Osteosarcoma of phalanges of hand is extremely rare. Often there is a delay in diagnosis because of patient delay and mildness of symptoms, or because of misdiagnosis at presentation. Osteosarcomas of hand have been reported to behave less aggressively than those arising in other sites.

A constant awareness for unusual lesions in unusual locations is important so that proper and prompt treatment can be provided. The prognosis once treated appropriately is excellent.

\section{Conflict of Interest: None}

\section{References}

1. Anningo KJ, Picci P, Fiocco et al. Osteosarcoma of the hands and feet: A distinct clinico-pathological subgroup. Virchows Arch 2013; 462: 109-20.

2. Marcuzzi A, Maiorana A, Adani R et al. Osteosarcoma of the scaphoid. J Bone Joint Surg 1996; 78-B: 699-701.

3. MA Mohd-Ariff et al. Phalangeal osteosarcoma mistaken for tuberculosis: A case report. Malaysian Orth J 2015; 9: 38-40.

4. Janu $\mathrm{A}$, Jain $\mathrm{N}$ et al. Radiological review of extremity osteosarcoma. J Bone Soft Tissue Tumors 2016; 2(1): 13-18.

5. Malhas AM, Sumanthi VP et al. Low Grade Central Osteosarcoma: A Difficult Condition to Diagnose. Hindwani Publishing Corporation 2012; 764-96.

Date of Submission: 2017-08-17 Date of Acceptance: 2017-08-18 